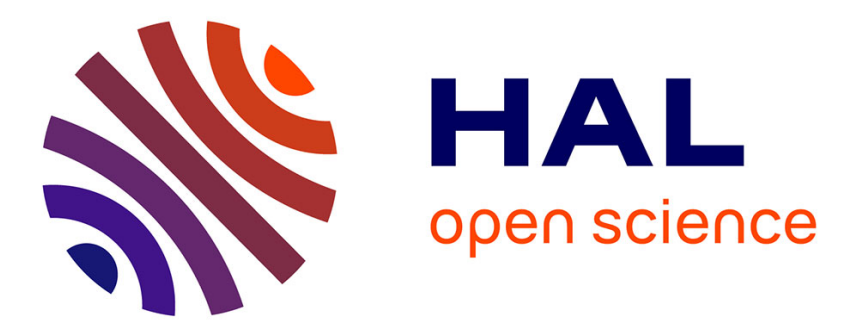

\title{
Low-Energy Electron Diffraction (LEED) Study of an Aperiodic Thin Film of Cu on 5-fold i-Al-Pd-Mn
}

\author{
Katariina Pussi, Dennis Reid, Nicola Ferralis, Ronan Mcgrath, Thomas
}

Lograsso, Amy Ross, Renee Diehl

\section{To cite this version:}

Katariina Pussi, Dennis Reid, Nicola Ferralis, Ronan Mcgrath, Thomas Lograsso, et al.. Low-Energy Electron Diffraction (LEED) Study of an Aperiodic Thin Film of $\mathrm{Cu}$ on 5-fold i-Al-Pd-Mn. Philosophical Magazine, 2008, 88 (13-15), pp.2103-2110. 10.1080/14786430701832412 . hal-00513853

\section{HAL Id: hal-00513853 \\ https://hal.science/hal-00513853}

Submitted on 1 Sep 2010

HAL is a multi-disciplinary open access archive for the deposit and dissemination of scientific research documents, whether they are published or not. The documents may come from teaching and research institutions in France or abroad, or from public or private research centers.
L'archive ouverte pluridisciplinaire HAL, est destinée au dépôt et à la diffusion de documents scientifiques de niveau recherche, publiés ou non, émanant des établissements d'enseignement et de recherche français ou étrangers, des laboratoires publics ou privés. 


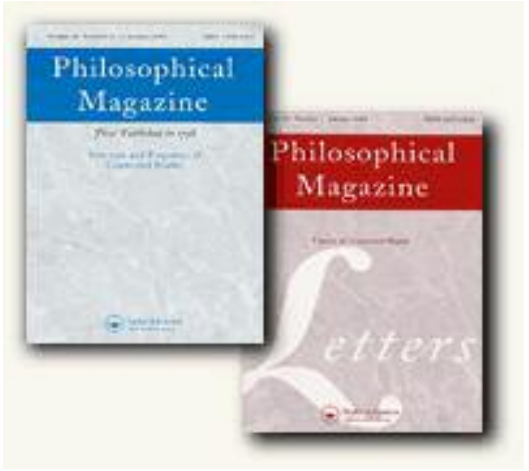

\section{Low-Energy Electron Diffraction (LEED) Study of an Aperiodic Thin Film of $\mathrm{Cu}$ on 5-fold i-Al-Pd-Mn}

\begin{tabular}{|r|l|}
\hline Journal: & Philosophical Magazine \& Philosophical Magazine Letters \\
\hline Manuscript ID: & TPHM-07-Oct-0277.R1 \\
\hline Journal Selection: & Philosophical Magazine \\
\hline Author: & 12-Nov-2007 \\
\hline Complete List of Authors: & $\begin{array}{l}\text { Pussi, Katariina; Lapennranta University of Technology, Electrical } \\
\text { Engineering } \\
\text { Reid, Dennis; University of Liverpool, Surface Science Research } \\
\text { Centre } \\
\text { Ferralis, Nicola; Penn State University, Physics Department } \\
\text { McGrath, Ronan; The University of Liverpool, Physics } \\
\text { Lograsso, Thomas; Iowa State University, Ames Laboratory } \\
\text { Ross, Amy; Iowa State University, Ames Laboratory } \\
\text { Diehl, Renee; Penn State University, Physics Department }\end{array}$ \\
\hline Keywords: & adsorption, copper, quasicrystals, thin films \\
\hline Keywords (user supplied): & adsorption, copper, quasicrystals \\
\hline TelAviv_revised.tex &
\end{tabular}

\section{S) ScholaroNE \\ Manuscript Central}




\title{
Low-Energy Electron Diffraction (LEED) Study of an Aperiodic Thin Film of Cu on 5-fold i-Al-Pd-Mn
}

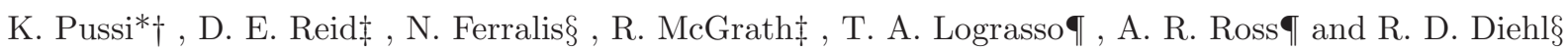 \\ $\dagger$ Lappeenranta University of Technology, Department of Electrical Engineering, P.O. Box 20 FIN-53851 \\ Lappeenranta, Finland \\ $\ddagger$ Surface Science Research Centre and Department of Physics, University of Liverpool, Liverpool, L69 3BX, UK \\ $\S$ Physics Department, Penn State University, University Park, PA 16802, USA \\ I Ames Laboratory, Iowa State University, Ames, IA 50011, USA \\ (Received 00 Month 200x; in final form 00 Month 200x)
}

\begin{abstract}
Thin films of copper grown on 5-fold i-AlPdMn at room temperature consist of domains that are rotationally aligned with the five primary symmetry directions of the substrate and which have one-dimensional aperiodic order. This aperiodic order is evident in scanning tunneling microscopy (STM) images as wide and narrow rows that are spaced according to a Fibonacci sequence. A low-energy electron diffraction (LEED) study of this film indicates that the structure within the domains is periodic along the rows, with a repeat distance equal to the nearest-neighbor separation in bulk Cu. To determine the complete structure, a dynamical LEED experiment was performed for a 5-layer $\mathrm{Cu}$ film at a sample temperature of $85 \mathrm{~K}$. The analysis was performed using two different computational methods, one based on quasicrystalline slabs and the other on periodic approximants. Of the model structures tested, the film is found to be most consistent with a structure based on the $\mathrm{Cu}\{100\}$ surface structure, but having aperiodic displacements, both in-plane and out-of-plane, along a $<110>$ direction.
\end{abstract}

\section{Introduction}

The growth of metal thin films on quasicrystalline substrates has led to the discovery of unique structures, many of which have nanoscale quasiperiodic ordering $[1,2]$. An interesting example of a nominally flat film having 1-dimensional quasiperiodic order is copper on the five-fold surface of $\mathrm{i}-\mathrm{Al}_{70} \mathrm{Pd}_{21} \mathrm{Mn}_{9}$. This structure was discovered by J. Ledieu et al. using scanning tunneling microscopy (STM) performed at room temperature [3]. At intermediate coverages (3-25 layers), the copper film forms ordered domains having five different orientations on the substrate. Within each domain, a quasiperiodic row structure is observed. The rows have two different separations $\left(\mathrm{S}=4.5 \mathrm{~A}^{\circ} \pm 0.2 \mathrm{~A}\right.$ and $\left.\mathrm{L}=7.3 \mathrm{~A}^{\circ} \pm 0.3 \mathrm{~A}^{\circ}\right)$ that are related approximately by the golden mean $(1.618 \ldots)$ and arranged in quasiperiodic order, which was identified as a part of Fibonacci sequence. Within the domains, a step height of $1.9 \AA$ was measured between the layers. Additional structural information was obtained by LEED measurements carried out at $85 \mathrm{~K}[2,4]$. These measurements indicated that copper atoms are periodically organized along the aperiodically spaced rows. The nearest-neighbor copper distance was measured to be $2.5 \pm 0.1 \AA$. Based on the nearest-neighbor distance and step height (from the STM study) a model consisting of strips of fcc $\{100\}$ copper was proposed. A more detailed characterization of this same film structure was carried out using Medium-Energy Ion Scattering (MEIS) [5]. In this study, the same kind of fcc $\{100\}$ strip structure was adopted as the model. The best-fit structure comprised an L strip having five atomic rows with $\mathrm{L}=$ $7.397 \AA$ and an $\mathrm{S}$ strip having three atomic rows with $\mathrm{S}=4.561 \AA$. The copper separation perpendicular to the strip pattern was fixed at its bulk value $(2.55 \AA)$, the nearest-neighbor copper distance was optimized along the strips to $2.71 \pm 0.02 \AA$, and the interlayer spacing was $1.67 \AA$.

\footnotetext{
* Corresponding author: Tel. +358-5 621 6771, Fax. +358-5-621 6799, E-mail katariina.pussi@lut.fi

Philosophical Magazine

ISSN 1478-6435 print/ISSN 1478-6443 online (c) 200x Taylor \& Francis

http://www.tandf.co.uk/journals

DOI: $10.1080 / 1478643 \mathrm{YYXxxxxxxx}$
} 
The sample used in this experiment $\left(\mathrm{Al}_{70} \mathrm{Pd}_{21} \mathrm{Mn}_{9}\right)$ was the one used in a previous study [4]. After insertion into ultrahigh vacuum, the sample preparation included of cycles of Ar+ ion sputtering and annealing to $940 \mathrm{~K}$ for a total of 20 hours. The temperature of the sample was measured by an infrared optical pyrometer (emissivity set to 0.35). The $\mathrm{Cu}$ source used in the experiment consisted of a tantalum wire $0.125 \mathrm{~mm}$ tightly twisted around a rod of copper (99.9\%, oxygen free). The sample temperature was $308 \mathrm{~K}$ during dosing. The $\mathrm{Cu}$ coverage, estimated using a comparison of Auger electron spectra and LEED patterns to those established in earlier experiments $[3,4]$ is 5-6 monolayers. The normal incidence LEED data were acquired at a surface temperature of $85 \mathrm{~K}$. The LEED measurements were performed using a conventional VG 3-grid rear-view LEED system. The data were collected using a CCD camera interfaced to a PC via a frame grabber board. The integrated diffraction spot intensities were extracted from the frames by integrating over a circle centered on the spot and subtracting a background intensity equal to the average of the intensity at the perimeter of the circle. The LEED intensities thus obtained are shown in Figure 1.

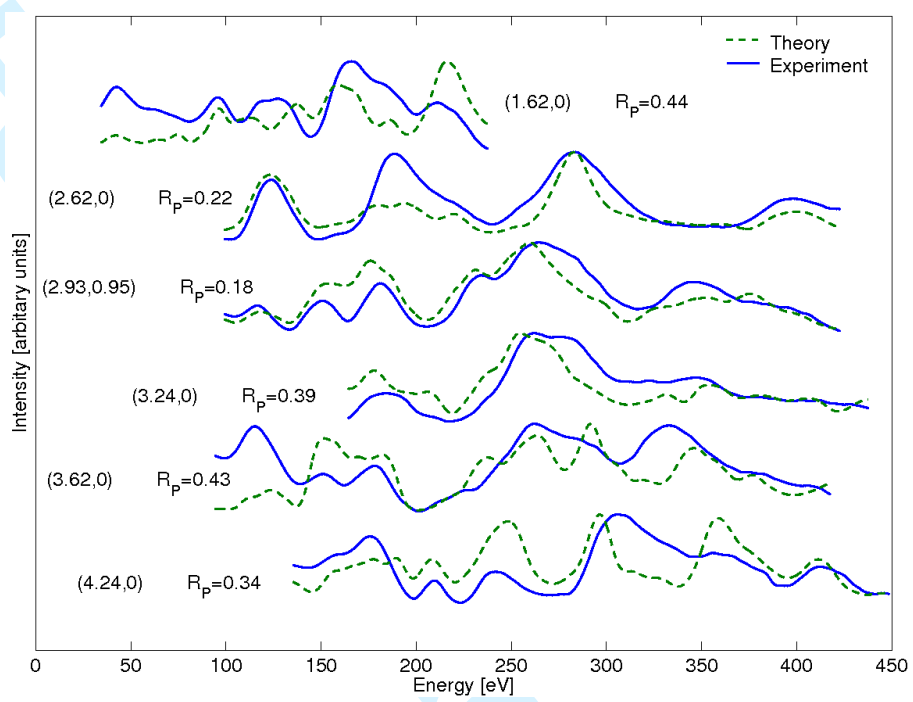

Figure 1. The LEED spectra consists of six symmetrically inequivalent beams. Solid lines correspond to the experimental beams and dashed lines correspond to the theoretical beams. Beams are labelled with k-vectors. The theoretical spectra corresponds to the best fit model $(\mathrm{Cu}\{100\}$ based periodic approximant model with $\mathrm{L}=3$ and $\mathrm{S}=2)$. Pendry $\mathrm{R}$-factors for each beam are shown.

\section{Calculations}

The analysis of the LEED intensities requires the calculation of intensities for model structures. Two approaches have been used for the calculation of intensities for quasicrystalline structures. One (quasiperiodic slab - method) is to use a quasiperiodic structure model and carry out a calculation with severe approximations for the positions of the atoms. The other (periodic approximant - method) is to use a periodic structure model that approximates the structure at short range but includes exact atom positions. We have recently compared these approaches for the decagonal AlNiCo surface [6].

The LEED calculations were done using two different programs; the Symmetrized Automated TensorLEED package of M.A. Van Hove et al. [7] (models based on periodic approximants) and the modified version of the W. Moritz's LEED program $[8,9]$ (models based on quasiperiodic slabs). Phase shifts for copper are calculated using the Phase shift program by A. Barbieri et al. [10] and the Pendry R-factor [11] is used for measuring the level of agreement between the experimental and the theoretical data. The energy range for the theoretical calculation was $20-460 \mathrm{eV}$ and the cumulative energy range is about 1700 $\mathrm{eV}$ which allows optimization of about 25 parameters. These adjustable parameters consist of the vertical positions of the atoms and the real part of the inner potential. The substrate AlPdMn is ignored in all 
calculations. This is justified by the fact that due to the thickness of the copper film, its contribution to

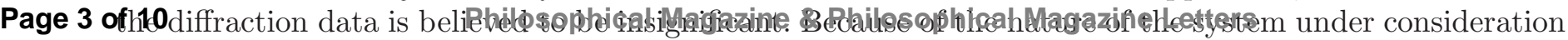
the number of possible model structures is almost unlimited, thus this paper will concentrate on those models we have considered the most promising among many other candidates.

The step height measured in the STM study $(1.9 \mathrm{~A})[3]$ is closest to the interlayer spacing of copper $\{100\}(1.81 \AA$ ). We have also considered the possibility of copper strips consisting of fcc $\{111\}$ and fcc $\{110\}$ arrangements (the interlayer spacings for those two are $2.08 \AA$ and $1.28 \mathrm{~A}$ respectively). Consistent with the prior STM/LEED study, the R-factor analysis prefers the structures built of fcc $\{100\}$ strips.

Calculations based on quasiperiodic slabs (Moritz's LEED program) are used as more global approach to finding preferred structures. More detailed calculations are done using the "periodic approximant-method" (Symmetrized Automated Tensor-LEED program).

\subsection{Models based on quasiperiodic slabs}

Quasiperiodic models are built of using strips of either $\{100\},\{111\}$ of $\{110\}$ copper. The L strip is $7.3 \AA$ wide and about $120 \AA$ long. The $\mathrm{S}$ strip is $4.5 \AA$ wide and about $120 \AA$ long. These strips are arranged to form a part of Fibonacci sequence LSLLSLSLLS. . in order to form layer with an area of about $120 \AA \times$ $120 \AA$. These layers are stacked to form a slab of thickness of about $15 \AA$. The stacking of the layers follows the stacking of either $\{100\},\{111\}$ or $\{110\}$ copper. The interlayer spacing is initially kept the same as for bulk $\mathrm{Cu}(1.81 \AA / 2.08 \AA / 2.28 \AA$, for $\{100\},\{111\}$ and $\{110\}$ respectively). The nearest-neighbor distance between atoms within the strips is kept at the bulk value $(2.55 \mathrm{~A})$. On the border of two strips this distance may be smaller or larger than bulk value, depending on the model.

The first model based on $\{100\}$ strips was built using three atom rows for L strip and two atom rows for S strip. In the preliminary calculations all layers were kept flat and the interlayer spacings were kept fixed. Only the real part of the inner potential was allowed to vary at this stage. These unrelaxed structures are referred to from now on as "bulk" structures. The top view of this model is shown in Figure 2 A. Closely looking at the figure, one can notice a problem with this model. The outermost atoms between the L strip in the first layer and the S strip in the second layer (or equally between the S strip in the first layer and the L strip in the second layer) are too close to each other. The distance between these atoms is about 1.9 A. To avoid this problem two modifications of this model were made. The first modification (Figure 2 B) has only one row of atoms in the $\mathrm{S}$ strips (instead of two). The second modification has two rows in both L and S strips (Figure $2 \mathrm{C}$ ).

Figure 3 shows top views of the models based on $\{111\}$ and $\{110\}$ strips. The model based on the $\{111\}$ strip has three atoms in the L strips and two atoms in the S strips. In the $\{110\}$ based model there are two atoms in the $\mathrm{L}$ strips and one atom in the $\mathrm{S}$ strips.

In the studies presented here, the interlayer spacings of the top seven layers were relaxed for the above mentioned models (in case of $\{100\}$-based models, this is equal to about $10 \AA$ ). The Pendry R-factors for the "bulk" structures and for the relaxed structures are listed in Table 1.

\subsection{Models based on periodic approximants}

The method of using periodic approximants to describe a quasiperiodic surface is explained in our earlier work [6]. Periodic models are build using a unit cell that consists of three L strips and two S strips. The L strip is initially $7.3 \AA$ and the $\mathrm{S}$ strip $4.5 \AA$ wide. These values are adjusted at a later stage to give optimum agreement. The $\mathrm{Cu}-\mathrm{Cu}$ distances within the strips have the bulk value $(2.55 \mathrm{~A})$ but on the border of two strips this may vary depending on the model. The L and S strips are arranged such that one unit cell = LSLSL. The dimensions of the unit cell are initially $30.9 \AA \times 2.55 \AA$. The interlayer spacing is initially 1.81 A which is the bulk value for $\mathrm{Cu}\{100\}$.

In the first model the L strip has three atoms in each layer and the S strip has two atoms in each layer, which makes 13 atoms in each layer of the unit cell. Models with and without a half atom diameter shift $(1.28 \mathrm{~A})$ between the $\mathrm{L}$ and the $\mathrm{S}$ strips were explored. Because LEED is not very sensitive to lateral 
A Philosophical Magazine \& ${ }^{B}$ hilosophical Magazine Letters
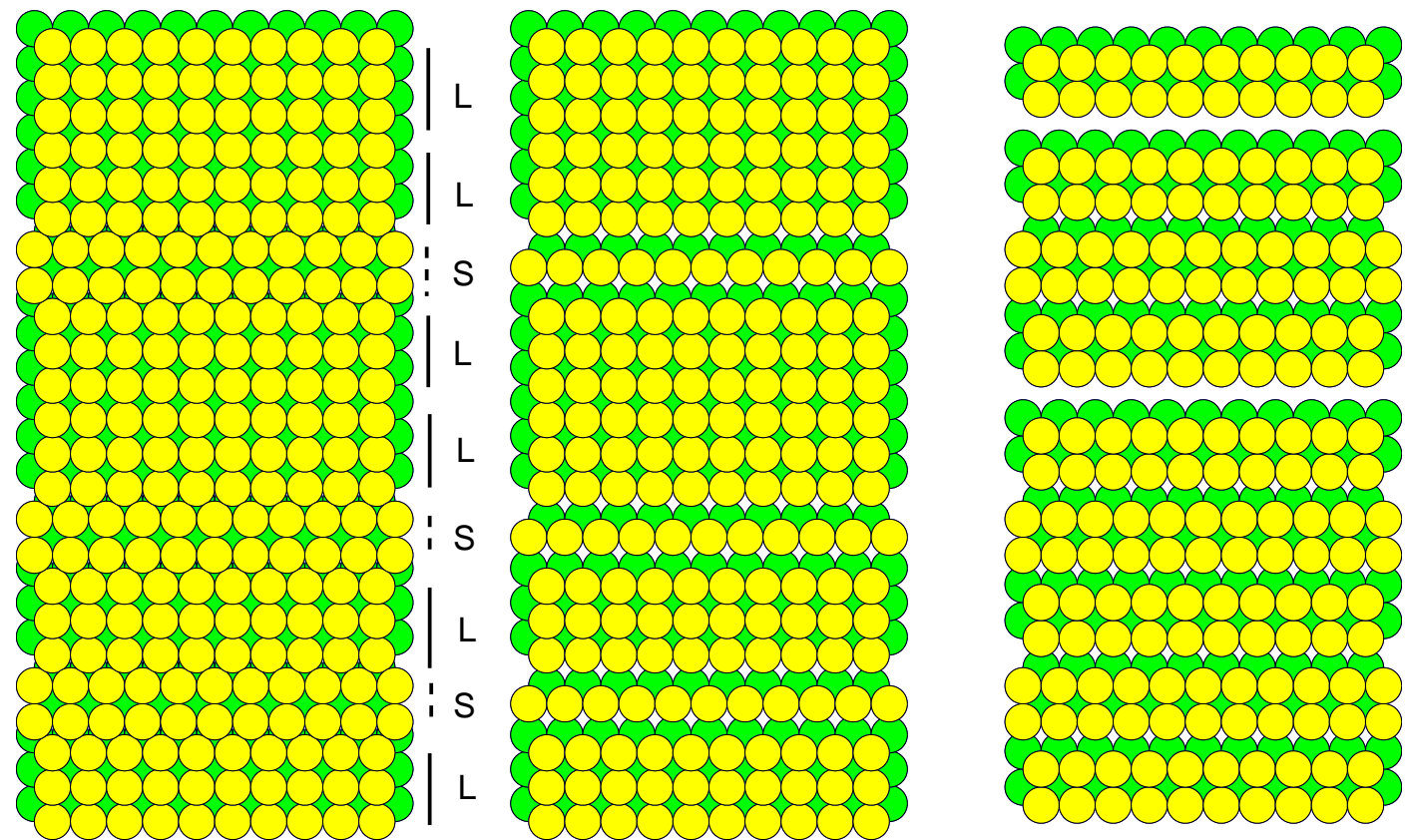

Figure 2. Top views of the three different variations of a quasiperiodic slab based on $\{100\}$ strips. In all A-C a $20 \AA \times 60 \AA$ part of the slab is shown (top two layers). A: Slab with the $\mathrm{L}=3$ atoms and the $\mathrm{S}=2$ atoms. $\mathrm{B}: \mathrm{L}=3$ atoms and $\mathrm{S}=1$ atom. C: $\mathrm{L}=2$ atoms and $\mathrm{S}=2$ atoms. The $\mathrm{L}$ and $\mathrm{S}$ sequence is same for all the models.
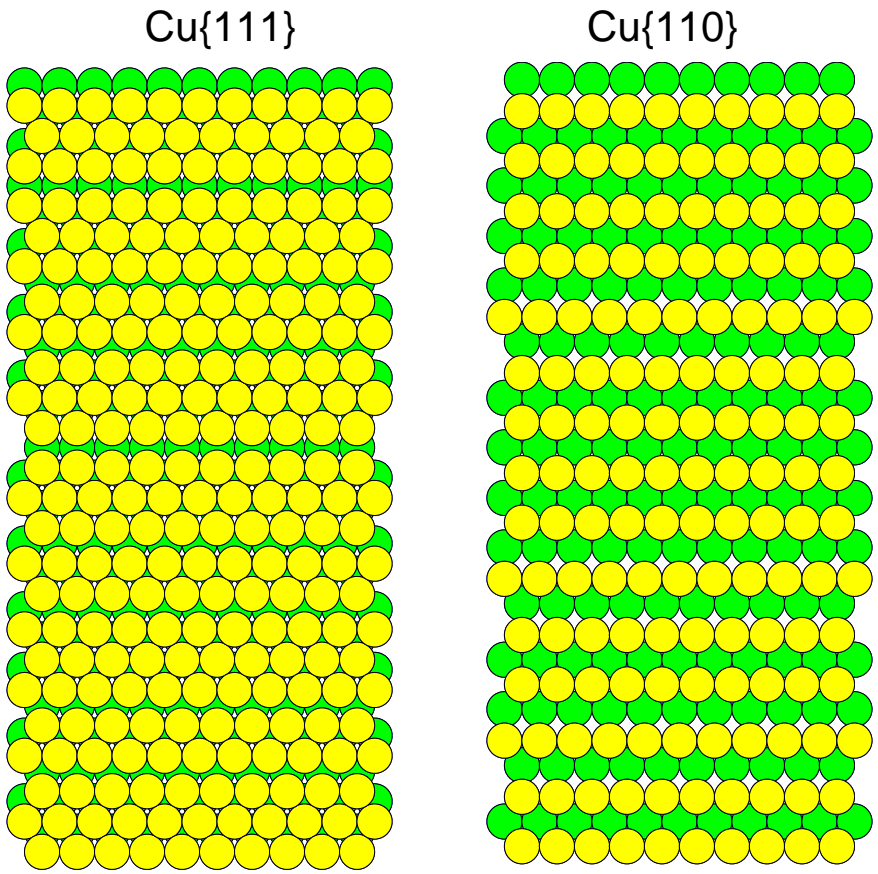

Figure 3. The top views of quasiperiodic slabs based on $\{111\}$ and $\{110\}$ strips. A $20 \AA \times 60 \mathrm{~A}$ part of the slab is shown (top two layers) for both crystallographic faces. The L and S sequence is same as in Figure 2.

parameters, this shift does not affect the $\mathrm{I}(\mathrm{E})$ curves much. Top and side views of this model (with the half atom diameter shift) are shown in Figure $4 \mathrm{~A}$. As discussed earlier with the quasiperiodic slab models, this model has one potential fault, which is the short nearest-neighbor distance.

The next modification of the model had alternating numbers of atoms in the $\mathrm{L}$ and the $\mathrm{S}$ strips in 
Page 5 of 10 ten atoms in every other layer, depending on how the atoms are arranged. Each of these variations has two possible terminations which makes the total number of models for this modification equal to four. One of these models $(\mathrm{L}=3$ in the first layer and 2 in the second layer, $\mathrm{S}=1$ in the first layer and 2 in the second layer) is show in Figure 4 B. These models all have reasonable nearest-neighbor copper distances. Since the STM observations indicate similar structures for all terraces, models such as these would require a significant dynamic rearrangement during the growth, so that the same type of layer is always present at the surface.
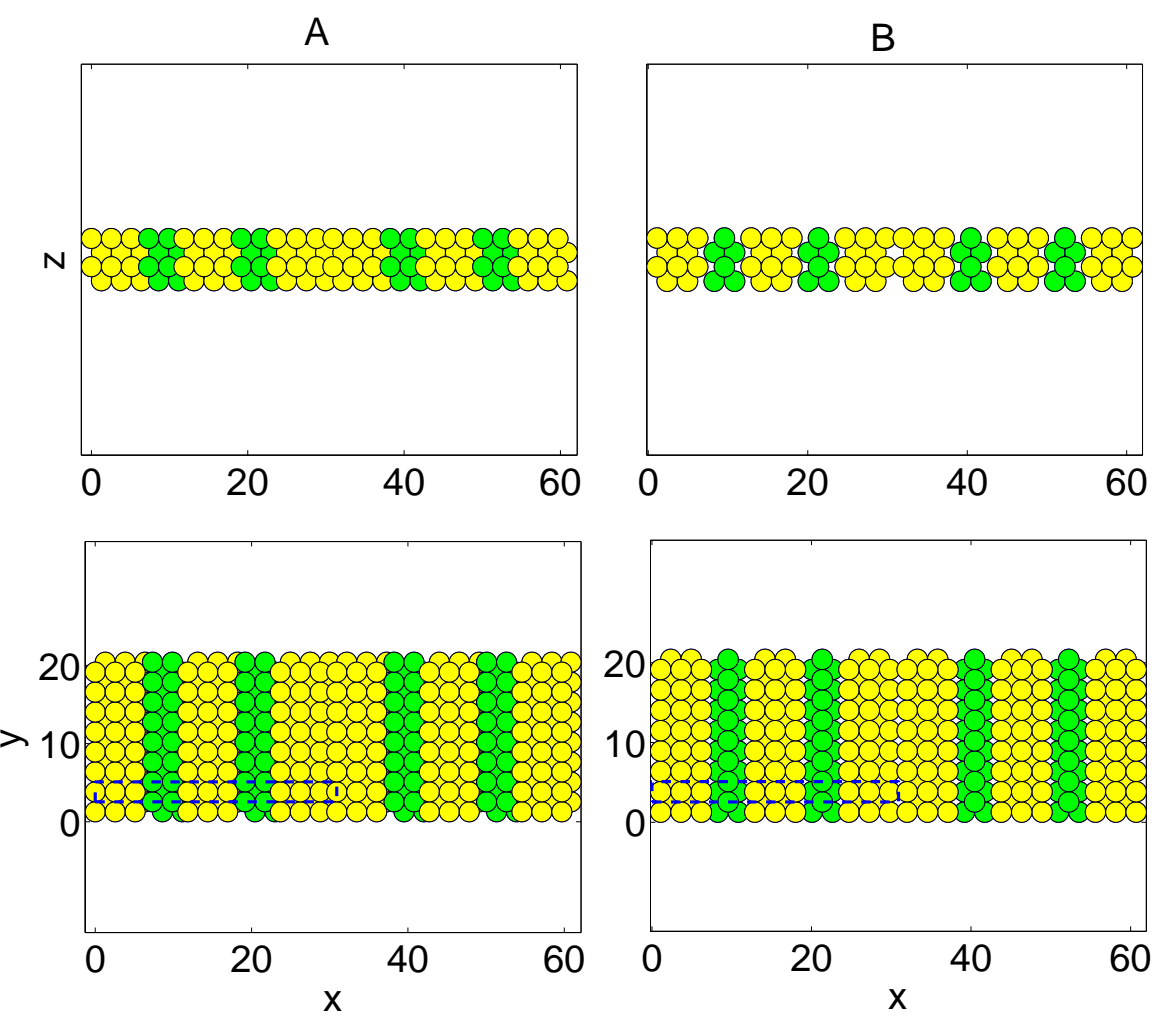

Figure 4. Top and side views of two "periodic approximant" - models. A: $\mathrm{L}=3$ atoms and $\mathrm{S}=2$ atoms. B: $\mathrm{L}=3$ or 2 atoms and $\mathrm{S}=$ 2 or 1 atom(s). Unit cell is shown with dashed line. Units are in angstroms.

Similarly to the quasiperiodic slab calculations, models in which $\mathrm{L}=3$ atoms and $\mathrm{S}=1$ atom, and in which both the $\mathrm{L}=\mathrm{S}=2$ atoms were tried. These models have 11/10 atoms in the unit cell in every layer and are very similar to the first periodic model except that there are two/three atoms fewer in the unit cell, making more room for the atoms and solving the problem of too short nearest-neighbor distance.

A metastable phase of bcc $\mathrm{Cu}$ is reported in the literature [12]. On $\mathrm{Au}\{100\}$, a thin $\mathrm{Cu}$ film forms a bcc structure while an orthogonal bct structure is observed at higher coverages [13]. Therefore we also considered a stepped orthorhombic structure with $\mathrm{a}=2.88 \AA$ in the aperiodic direction and $\mathrm{b}=2.5 \AA$ in periodic direction. With an angle $\alpha=13.28^{\circ}$ with respect to the $\{100\}$ surface, this stepped surface is one-dimensionally quasiperiodic with $\mathrm{L}=7.34 \AA$ and $\mathrm{S}=4.56 \AA$. Because of the small interlayer spacing of this stepped structure, smaller unit cell of LSL was used. With this unit cell, 25 free parameters means optimizing down to about 5 A.

Table 1 shows the Penry R-factor for the relaxed structures. The number of relaxed parameters in each model is equal to about 25 (this number varies slightly depending on the model). The expansion of the $\mathrm{Cu}-\mathrm{Cu}$ spacing along the periodic direction, which was seen in the MEIS [5] study, was considered for all the models. The effect of this expansion was not systematic. For some of the models it slightly made the agreement better, for others the effect was the opposite. 


\begin{tabular}{|c|c|c|c|}
\hline Model & $\begin{array}{c}\mathrm{R}_{P} \\
\text { ("bulk" / relaxed) }\end{array}$ & $\begin{array}{c}\mathrm{R}_{P} \\
\text { erePbilssappisatimagta } \\
\text { (relaxed) }\end{array}$ & $\begin{array}{c}\text { iropltettens } \mathrm{Cu}-\mathrm{Cu} \text { distance } \\
\text { along the rows }\end{array}$ \\
\hline fcc $\{111\}$ & $0.93 / 0.87$ & - & - \\
\hline fcc $\{110\}$ & $0.92 / 0.81$ & - & - \\
\hline fcc $\{100\}$ & & & \\
\hline $\mathrm{L}=3 \& \mathrm{~S}=2$ & $0.85 / 0.75$ & 0.32 & $2.47 \AA$ \\
\hline $\mathrm{L}=3 \& \mathrm{~S}=1$ & $0.87 / 0.75$ & 0.44 & $2.55 \AA$ \\
\hline $\mathrm{L}=2 \& \mathrm{~S}=2$ & $0.81 / 0.74$ & 0.46 & $2.55 \AA$ \\
\hline $\mathrm{L}=3 / 2 \& \mathrm{~S}=2 / 1$ & - & 0.47 & $2.63 \AA$ \\
\hline $\mathrm{L}=2 / 3 \& \mathrm{~S}=2 / 1$ & - & 0.47 & $2.55 \AA$ \\
\hline $\mathrm{L}=3 / 2 \& \mathrm{~S}=1 / 2$ & - & 0.41 & $2.57 \AA$ \\
\hline $\mathrm{L}=2 / 3 \& \mathrm{~S}=1 / 2$ & - & 0.44 & $2.63 \AA$ \\
\hline Stepped bct & - & 0.39 & $2.59 \mathrm{~A}$ \\
\hline
\end{tabular}

Table 1. Table with the Pendry R-factors for different modles. For different variations of $\{100\}$ strips $\mathrm{L}=\mathrm{x} / \mathrm{y}$ means that $\mathrm{L}$ has $\mathrm{x}$ atoms in the first layer and $\mathrm{y}$ atoms in the second layer. Same applies for $\mathrm{S}=\mathrm{x} / \mathrm{y}$. For the "periodic approximant" - models the optimized Cu-Cu distance along the periodic direction is shown. The higher the R-factor value is, the larger is the uncertainty associated with that value. This means that large R-factor values provide no information to distinguish bad structures from each other.

\section{Conclusions}

In this paper we have presented new LEED data for aperiodic $\mathrm{Cu}$ film grown on i-AlPdMn. Based on these and earlier studies, we have proposed ten possible structure models and have performed extensive quantitative LEED analyses for the most promising ones. On the grounds of R-factor analysis, the models based on strips of $\{100\}$ copper are found to be the most promising of those studied. The best fit is for the model with $\mathrm{L}=3$ and $\mathrm{S}=2$, but it contains unphysical $\mathrm{Cu}$ spacings. The next best fit is for the stepped model. The relaxations in the other models also suggest a step-like surface relaxation. However none of the models gives a level of agreement comparable to studies of other clean $\mathrm{Cu}$ surfaces, which suggest that the exact details of the structure are yet to be determined. One factor which could affect these results is the potential for the electrons to penetrate the $\mathrm{Cu}$ film and scatter from the substrate. Although any such scattering is expected to be minor for a 5-6 layer $\mathrm{Cu}$ film, coverage-dependent measurements might be useful.

We gratefully acknowledge many useful and interesting discussions with Julian Ledieu, Joe Smerdon and Martin Gierer. This research was supported by The Academy of Finland (Project No. 204726) and NSF (Grant No. DMR0505160).

\section{References}

[1] V. Fournée and P.A. Thiel, J. Phys. D: Appl. Phys. 38, R83 (2005).

[2] H.R. Sharma, M. Shimoda and A.P. Tsai, Advances in Physics 56, 403 (2007).

[3] J. Ledieu, J. T. Hoeft, D. E. Reid, J. A. Smerdon, R. D. Diehl, T. A. Lograsso, A. R. Ross and R. McGrath, Phys. Rev. Lett. 92, $135507(2004)$

[4] J. Ledieu, J. T. Hoeft, D. E. Reid, J. A. Smerdon, R. D. Diehl, N. Ferralis, T. A. Lograsso, A. R. Ross and R. McGrath, Phys. Rev. B 72, 035420 (2005).

[5] J. A. Smerdon, J. Ledieu, R. McGrath, T. C. Q. Noakes, P. Bailey, M. Draxler, C. F. McConville, T. A. Lograsso and A. R. Ross, Phys. Rev. B 74, 035429 (2006).

[6] K. Pussi, N. Ferralis, S. Curtarolo, M. Gierer, M. Mihalkovic, M. Widom, C. J. Jenks, P. Canfield, I. Fisher and R. D. Diehl, Phys. Rev. B 73, 184203 (2006).

[7] M.A. Van Hove, W. Moritz, H. Over, P.J. Rous, A. Wander, A. Barbieri, N. Materer, U. Starke and G.A. Somorjai, Surf.Sci. Rep 19 (1993) 191.

[8] M. Gierer, M.A. Vanhove, A.I. Goldman, Z. Shen, S.-L. Chang, P.J. Pinhero, C.J. Jenks, J.W. Arderegg, C.-M. Zhang and P.A. Thiel, Phys. Rev. B 57, 13, (1998) 7628.

[9] W. Moritz, J.Phys.C: Solid State Physics, 13 (1984) 353.

[10] A. Barbieri and M.A. Van Hove, private communication.

[11] J.B. Pendry, J.Phys.C: Solid State Physics, 13 (1980) 937.

[12] Z. Tang, M. Hasegawa, Y. Nagai, M. Saito, Phys. Rev. B 65, 195108 (2002).

[13] B.M. Ocko, I.K. Robinson, M. Weinert, R. J. Randler and D. M. Kolb, Phys. Rev. Lett. 83, 780 (1999) 


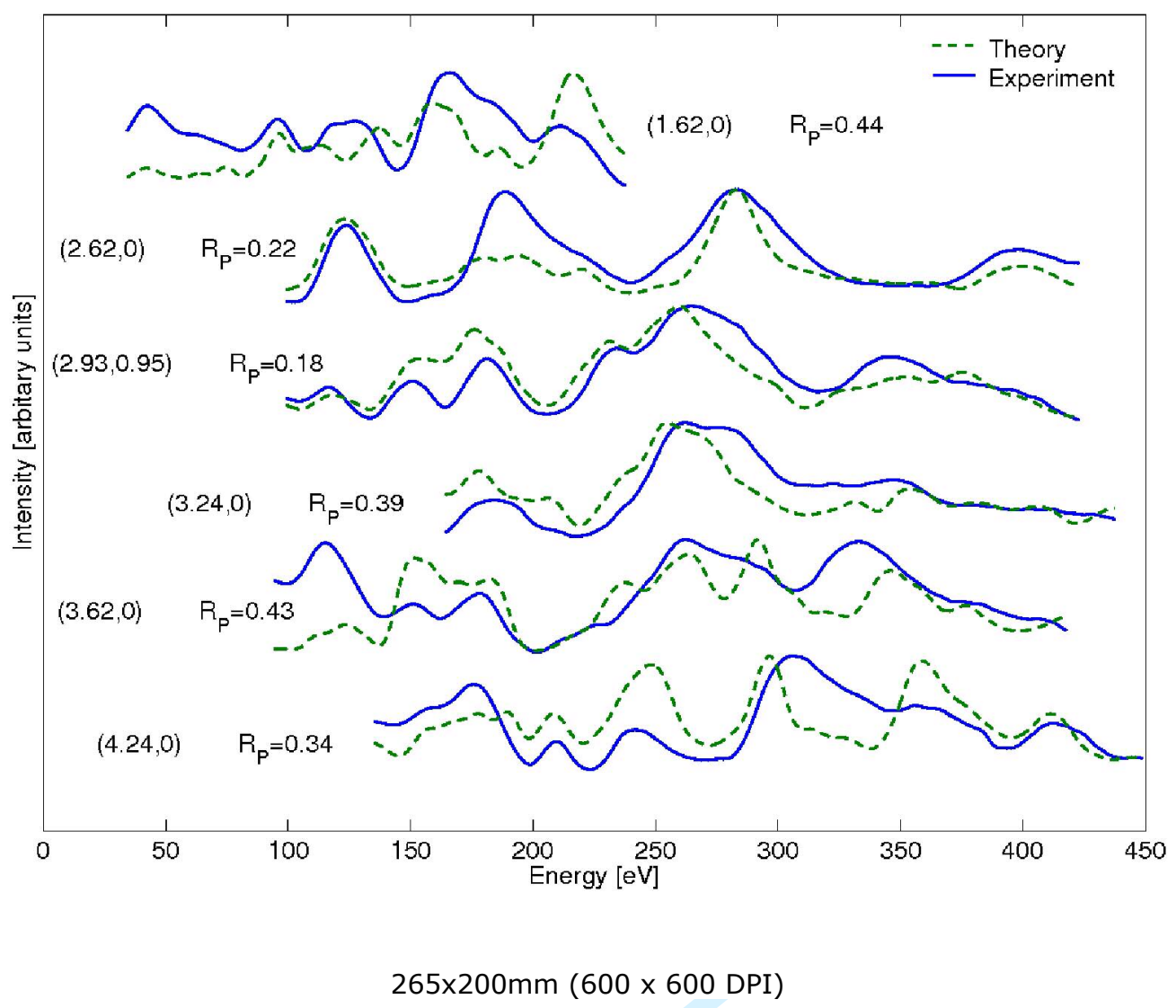

http://mc.manuscriptcentral.com/pm-pml 

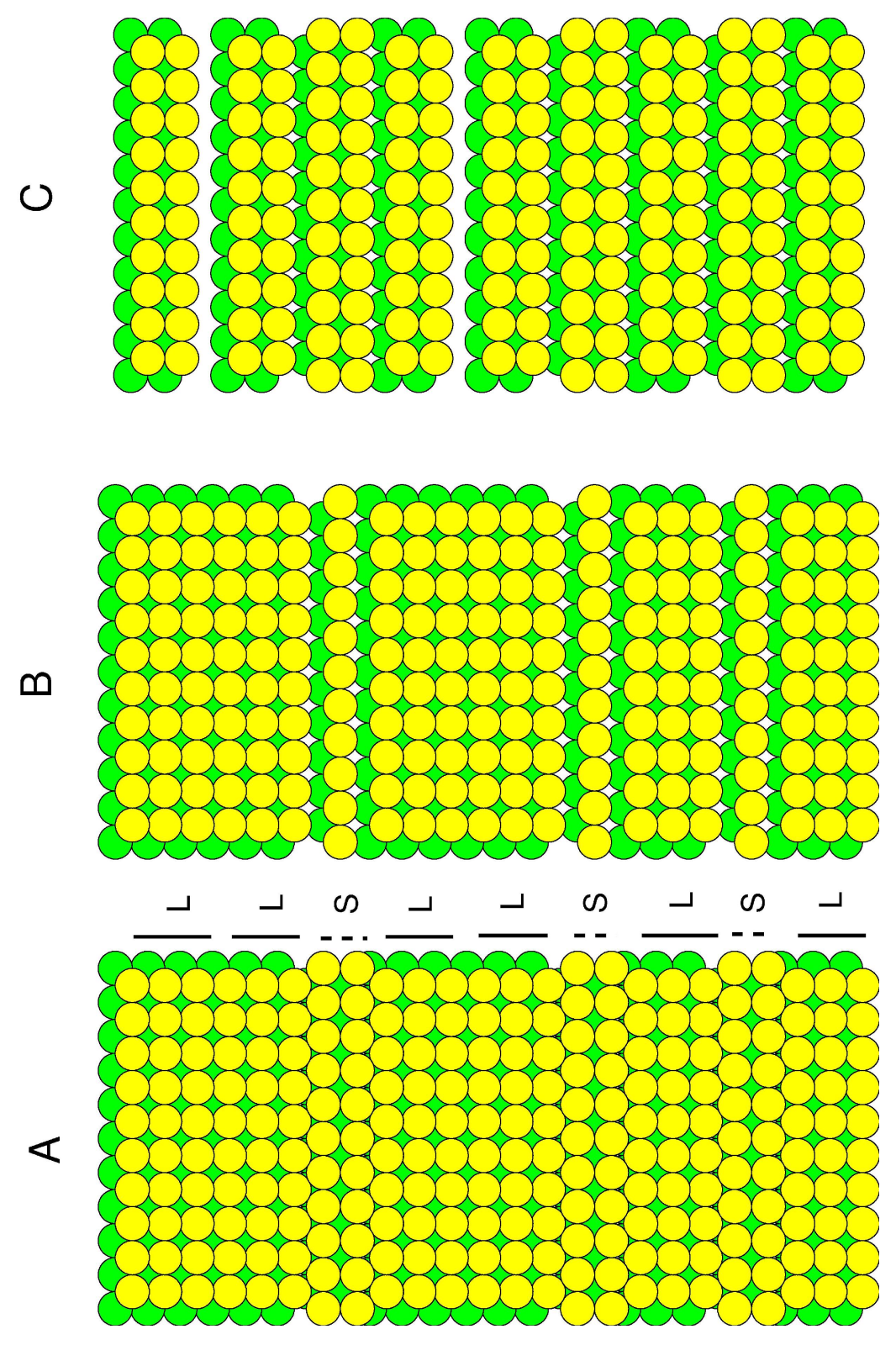

$176 \times 262 \mathrm{~mm}(600 \times 600 \mathrm{DPI})$ 

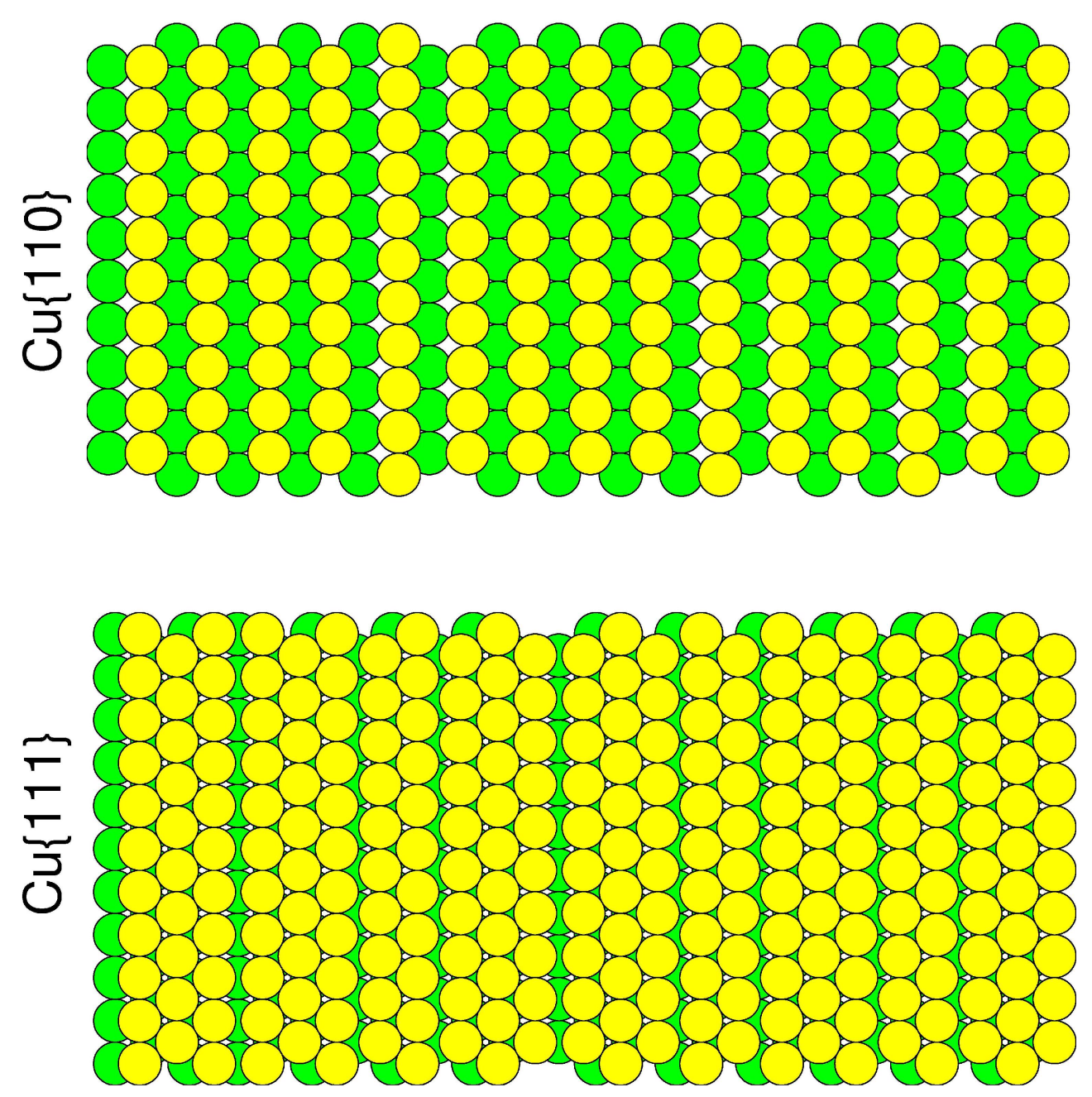

$177 \times 176 \mathrm{~mm}(600 \times 600 \mathrm{DPI})$ 

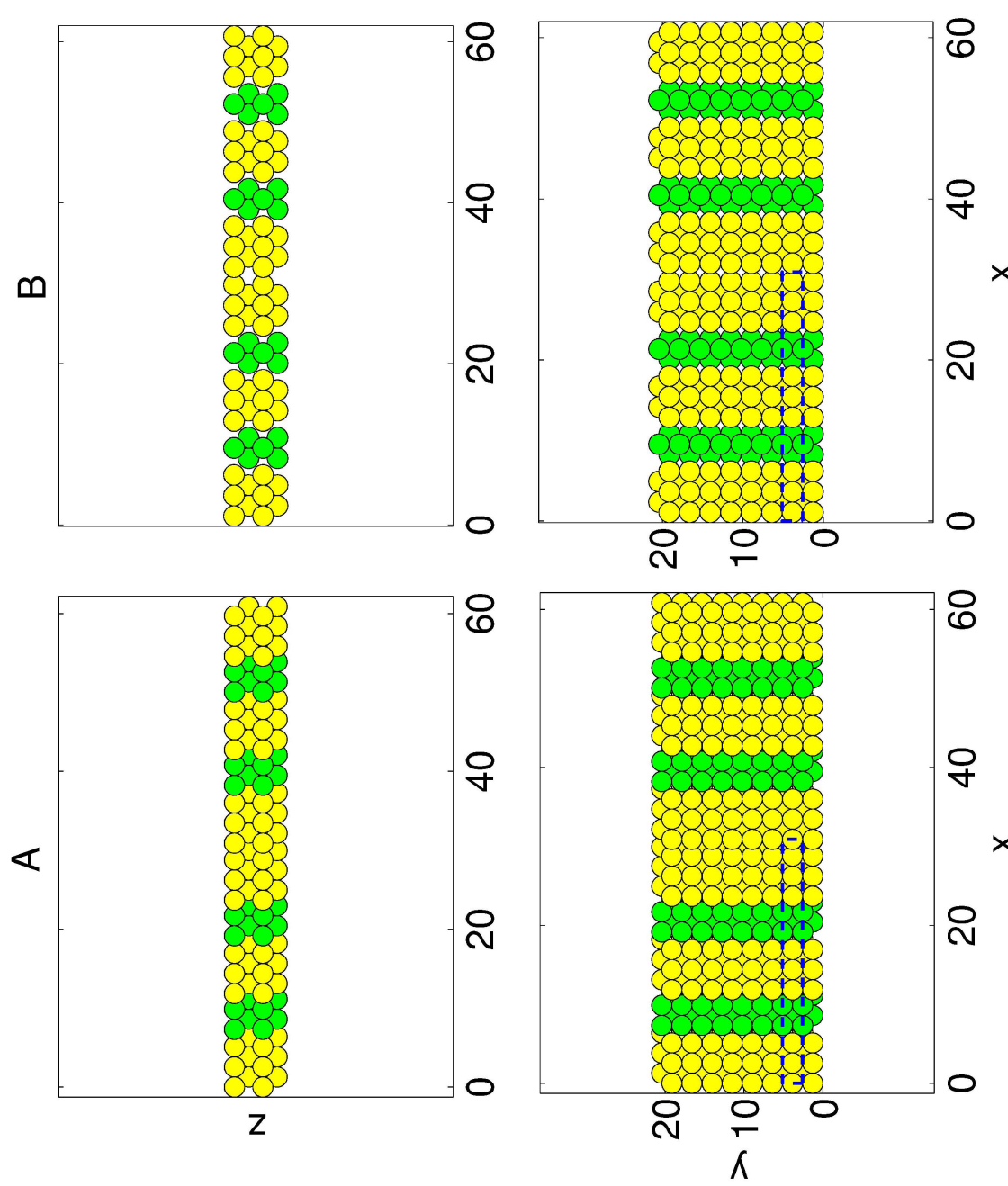

$232 \times 310 \mathrm{~mm}(600 \times 600 \mathrm{DPI})$ 